\title{
Social Media Engagement of Stakeholders: A Decision Tree Approach in Container Shipping
}

\section{Ebru SURUCU BALCI ${ }^{1-2}$, Gökcay BALCI ${ }^{3}$, Kum Fai YUEN ${ }^{4}$}

\author{
${ }^{1}$ School of Management, University of Bradford, Bradford, UNITED KINGDOM, \\ ebrusurucu.cbu@gmail.com \\ ${ }^{2}$ Faculty of Business, Manisa Celal Bayar University, Manisa, TURKEY \\ ${ }^{3}$ Huddersfield Business School, University of Huddersfield, Huddersfield, UNITED \\ KINGDOM, g.balci@hud.ac.uk \\ ${ }^{4}$ School of Civil and Environmental Engineering, Nanyang Technological University, \\ SINGAPORE, yuenkf@cau.ac.kr
}

\section{For citation:}

Surucu-Balci, E., Balci, G., \& Yuen, K. F. (2020). Social Media Engagement of Stakeholders: A Decision Tree Approach in Container Shipping. Computers in Industry, 115, 103152. https://doi.org/10.1016/j.compind.2019.103152

\begin{abstract}
Social media provides a significant avenue for stakeholder engagement which is crucial to ensure loyalty and satisfaction of stakeholders who possess valuable resources that can influence the business outcomes. Container lines - imperative members of global supply chains and facilitators of international trade - utilize social media to engage their stakeholders due to environmental and commercial complexity their business. However, not all social media posts generate the same amount of stakeholder engagement. This study aims to identify and examine the social media post characteristics that lead to higher stakeholder engagement in the container shipping market. The study applies Chi-Squared Automatic Interaction Detection method to categorize social media posts based on their engagement levels. The analysis is conducted on the tweets of four global container lines which are posted between 1 September 2018 and 31 January 2019. The results demonstrate that social media posts of container lines have varying effects on engagement level. We found that fluency of tweets, tangibility of company resources in the tweet, vividness level, content type, existence of a link, and existence of a call-to-action significantly influence the container lines' stakeholder engagement rate. This study is the first that finds out SM post classes based on the interaction between their characteristics and engagement rates by employing a decision tree methodology. The results are expected to help container lines in their social media management and stakeholder engagement policies.
\end{abstract}

Keywords: CHAID, Decision Tree, Shipping, Social Media, Stakeholder Engagement, Twitter

1. Introduction

In WEB 1.0 technology, stakeholders were able to access a rich amount of content by reading, listening, and watching. However, the communication happened in a monologue structure. 
Conversely, Web 2.0 Technology enabled stakeholders and organizations to interact with each other. Particularly, social media (SM) platforms have created an interactive communication environment for people and businesses. SM - a Web 2.0 technology - allows companies to build interactive communication at a very low cost with their stakeholders from around the world. The companies can reach out to a great number of stakeholders and organizations, and can have real-time conversations with them almost instantly and without physical barrier (Zhang and Lin, 2015).

Although some concerns are present related to the usage of WEB 2.0 technology in workplace (Faci et al., 2017), this disruptive development has been embraced and adopted by companies because the technology, particularly SM, can provide significant benefits and opportunities for companies. These benefits have also garnered the attention of academic research. For instance, Park et al. (2016) found that engagement in social network site increases firm value. Baird and Parasnis (2011) found that SM can be an effective tool for customer relationship management and help forge closer relationships with customers which can eventually increase revenues and reduce costs. The usage of SM by salespeople can also increase their responsiveness and customers;' satisfaction (Agnihotri et al., 2016). SM usage can also boost operational efficiency and innovativeness of organizations by facilitating communication with their stakeholders (Lam et al., 2016). Performance of a firm can also be improved via SM usage (Tajvidi and Karami, 2017). Besides, through SM, brand loyalty of customers can be increased (Erdoğmuş and Cicek, 2012; Gomez et al., 2019) and brand engagement of firms' employees can be improved (Pitt et al., 2017). Overall, SM helps companies to engage their stakeholders, which eventually presents various benefits for the companies.

Container lines have also adopted SM to engage their stakeholders and have received much benefits. Container lines are shipping companies with global operations challenged by pressures arising from increasing competition, environmental regulations, technological developments, legal and political issues, and organizational concerns (Yuen and Lim, 2016; Balci and Cetin, 2017. Container lines also faces business and environmental pressures from the supply chains such as performing better at sailing reliability, increasing safety, and reducing emissions (Yang et al., 2018). In such a challenging environment, container lines need to maintain constant communication and information exchange with their stakeholders to better perform in marketing, comply with local and international regulations, manage corporate social responsibility (CSR) activities, handle emergencies effectively, and increase brand reputation. Thus, utilizing SM is essential in container shipping to manage stakeholder relationship.

The benefits of being active on SM are well documented both in the literature and practice. However, SM engagement is a tricky issue that needs special attention and effort. The effect of SM posts on engagement rates is varied. For instance, some posts generate higher engagement rate than others. The engagement rate of a post was found to depend on several factors such as content type and media type (Cvijikj and Michahelles, 2013; Denktas-Sakar and Surucu, 2019). Hence, it is vital for container lines to identify the factors influencing the engagement rates and explore SM post classes that result in higher engagement rates. Consequently, the lines can formulate and apply a more effective SM management policy to engage their stakeholders. At present, despite the existence of SM studies in public transportation (Manetti et al., 2017), only 
a few studies have investigated SM in shipping (Katona and Sarvary, 2014; Buratti et al., 2018). However, to the authors' best knowledge, none has focused on the engagement model of shipping companies. At the tactical or operational level, there is presently very limited knowledge with regards to how dialogues and messages should be structured and presented to engage stakeholders.

Motivated by stakeholder theory, the objective of this study is to determine SM post groups which lead to higher stakeholder engagement. We apply chi-squared automatic interaction detection (CHAID), which is a decision tree method. Our findings demonstrate that a total of eight SM post characteristics influence the stakeholder engagement rate and significantly classify the posts based on their relationship with the engagement rate. The results of the study contribute to stakeholder engagement, SM literature, and the container shipping literature. In the following sections, we presented the literature review in which we reviewed the concept of stakeholder engagement and reviewed previous studies on B2B SM research and the determinants of stakeholder engagement through SM. Then, we presented the sections on methodology, results, discussion, and theoretical and practical implications.

\section{Literature Review}

\subsection{Stakeholder engagement}

Firms' motivation for using SM can be explained using stakeholder theory. Stakeholder theory rejects the separation thesis which proposes that business and ethics are independent (Freeman et al.; 2004). The theory opposes a single-objective view of the firm which focuses solely on its shareholders to achieve economic performance. Instead, stakeholder theory suggests that for firms to be sustainable in the long-run, they should seek to satisfy the needs of their stakeholders who include customers, employees, shareholders, authorities, suppliers, and the society (Yang, 2018; Yuen et al., 2018). Essentially, the theory posits transcending beyond the narrow focus of satisfying shareholders' needs but the needs of a wider constituent of parties who have a legitimate or silent interest in the company (Freeman, 1984; Yuen et al., 2017).

The reason for embracing the wider approach is grounded on the notion that these stakeholders possess valuable resources such as capital, knowledge, time, materials and effort which serve as input to a firm's production of goods and services (Freeman et. al.; 2004; Wolf, 2014). For instance, employees hold valuable knowledge and dedicate time and effort to plan, manage, and run the firm whereas suppliers provide materials for a firm to produce goods and services. Consequently, the resources possessed by stakeholders confer them the power to punish or reward a firm by adjusting these resources, depending on whether their needs are satisfied.

The ability of stakeholders to influence the business outcomes of firms has led to the practice of stakeholder engagement which is defined as the 'involvement of stakeholders in dialogue to determine their social, environmental and economic concerns about the firm to improve its decision-making and accountability' (Schaltegger and Stefan, 2017). The purpose of stakeholder engagement is to garner loyalty from stakeholders which can increase their commitment of resources to a firm. 
In general, a typical stakeholder engagement process (see Figure 1) comprises ten stages which begins with the (1) identification of stakeholders, (2) segmentation of stakeholder categories, (3) prioritization of stakeholders, (4) definition of levels of engagement, (5) review of channel of engagements, (6) design of engagement model, (7) identification of relevant issues, (8) identification of risks and opportunities, (9) design of action plan, and (10) monitor and report. These stages can be categorized into stakeholder map, engagement model, stakeholder concerns and action plan (Iberdrola, 2019).

The stakeholder map involves recognizing and categorizing the stakeholders of a firm. It also requires the prioritization of the stakeholder categories on the basis of their influence on a firm's sustainable performance. The engagement model necessitates deciding and setting a predefined level of engagement with stakeholders. It also involves examining the various modes of engagement and selecting suitable modes to support the level of engagement. The stakeholder concerns involve finding out the issues faced by each stakeholder categories and evaluating the risks and opportunities that arise from these issues. Finally, the action plan concerns the formulation of strategies to address the issues faced by stakeholders. It also includes the creation of a feedback system that evaluates and reports the effectiveness of the strategies to satisfy stakeholders.

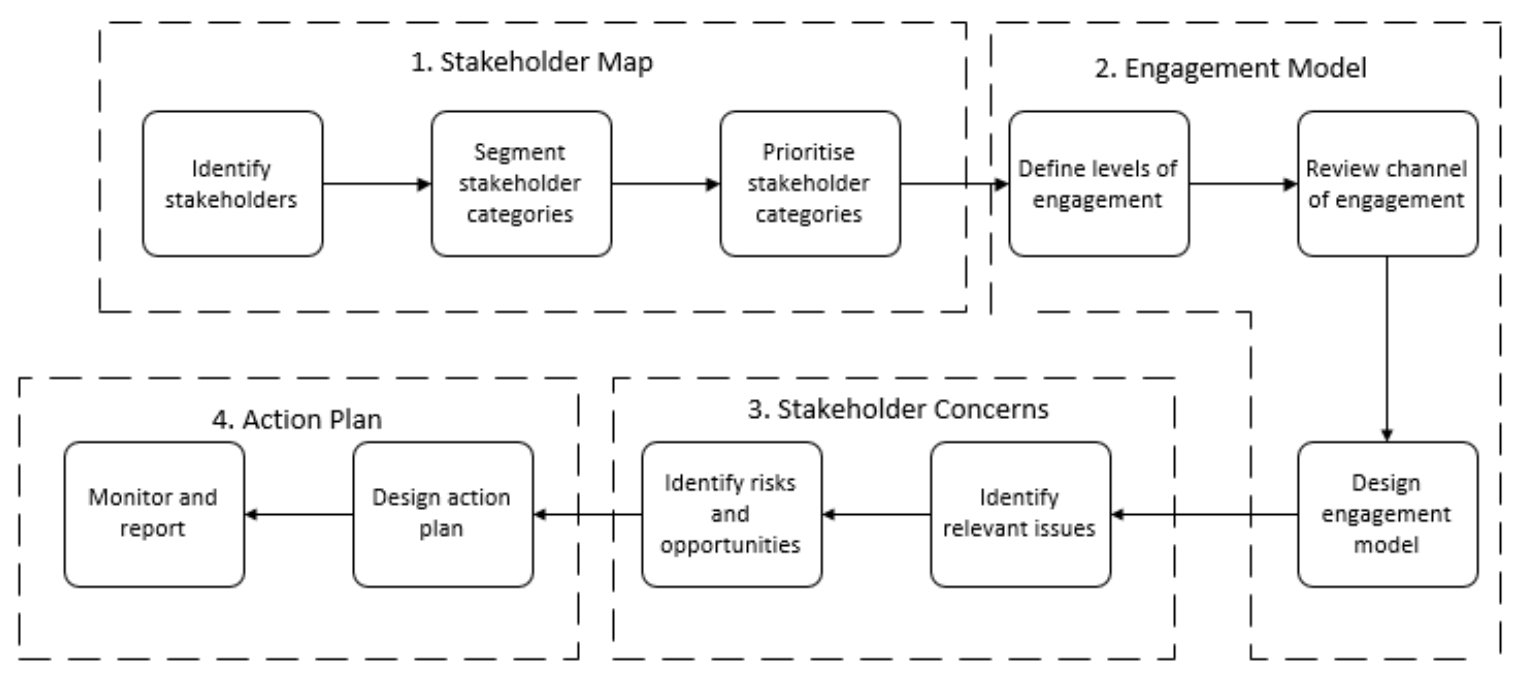

Figure 1. The stakeholder engagement process

Existing research on stakeholder engagement in the shipping context mainly relates to the categories on the stakeholder map and stakeholder concerns. For instance, there have been a few recent papers that discussed the stakeholders' conflicts and challenges in the development of ports and shipping services (Kuznetsov et al., 2015; Denktas-Sakar \& Karatas-Cetin, 2012). However, very little research has explored the engagement model of shipping companies. Particularly, at the tactical or operational level, there is presently very limited knowledge with regards to how dialogues and messages should be organized and presented to achieve the intended engagement from stakeholders. 
With regards to the category on engagement model, SM can be viewed as a channel of engagement to complement other traditional channels such as focus groups, interviews, meetings, sustainability reports, newspaper articles, and television.

\subsection{Social Media in B2B Industries}

The literature indicates that B2B companies lag behind consumer companies to adopt SM (Ammirato et al., 2018; Quinton and Wilson, 2016), and relevant academic research is mostly focused on the B2C context. Bernard (2016) conducted a survey study on SM marketing managers in $\mathrm{B} 2 \mathrm{~B}$ companies and found that they are not as prepared as their counterparts with regards to SM adoption. Nonetheless, many B2B companies in different industries have been utilizing SM platforms, and in fact, some of them, such as Maersk, are considered to be as successful as their B2C counterparts (Katona and Sarvary, 2014).

SM research in industrial markets has garnered a significant attention of many scholars especially since 2010 (Salo, 2017). This attention makes sense because B2B SM application differs from B2C and it needs special attention (Swani et al., 2014). For instance, Iankova et al. (2018) ascertained that B2B companies differ from B2C ones in terms of their purposes and perceived results of using SM, i.e. relationship building or attracting new customers (Iankova et al., 2018). Besides, each B2B industry is unique and the SM usage varies significantly among them (Ammirato et al., 2018). Hence, SM has become a popular research topic in B2B as well.

Early B2B researchers conduct survey studies and investigate the extent B2B companies use SM and their motivations. Jussila et al. (2014) found that, regarding customers, the most common motivations to use SM are marketing, communications, and employer branding and recruitment. Considering partners, the main reason is to communicate and collaborate with them. In general, B2B managers use SM mostly for improving company reputation, increasing customer interest, increasing customer awareness' enhancing customer relation, and supporting sales (Andersson and Wikström, 2017; Broekemier et al., 2015; Michaelidou et al., 2011).

Many empirical studies have also been conducted to examine the benefits of SM in B2B markets. Regarding marketing-related benefits, one of them is that SM enhances customer loyalty and satisfaction. SM usage by B2B salespeople significantly affects their responsiveness and improves customer satisfaction, and it also has a positive indirect effect on satisfaction and loyalty. (Agnihotri et al.,2016; Trainor et al., 2014)

SM can also positively influence sales in B2B context. SM utilization positively influences sales process and sales performance (Rodriguez et al., 2012: Schulz et al., 2012). Foltean et al. (2018) ascertained that SM can directly influence customer relationship management capabilities and indirectly affect a firm's performance in market, sales, and profitability growth. Customer service can also be improved through SM (Moore et al., 2013). Furthermore, SM in B2B can enhance branding strategies of companies (Brennan and Croft, 2012; Cawsey and Rowley, 2016).

Apart from marketing purposes, SM helps in solving intra-organizational issues faced by B2B companies. For instance, Pitt et al (2017) suggested that SM can play a significant role in 
employees' brand engagement. Dreher (2014) suggests that employees of a firm act as an ambassador of the firm through SM and contribute to reputation of the brand. In addition, SM can also be used as an effective tool for recruiting purposes (Andersson and Wikström, 2017; Fisher et al., 2014).

SM can also facilitate the internal and external communication of a firm, which, eventually, increases operational efficiency and innovativeness (Lam et al., 2016). Cheng and Krumwiede (2018) stated that SM use can positively enhance the supplier involvement in new product development. Wang et al. (2016) found that SM capabilities of B2B firms enhance the communication, which improves business performance in marketing, innovation, and collaboration. Drummond et al. (2018) indicate that SM improves B2B relations and networks which helps in information search, collaboration, operational process coordination, and reconfiguration processes. According to Steyn et al. (2010), SM usage can also assist in the effective management of public relations.

As the literature suggests, the benefits of SM use in B2B markets are diverse, and it can help firms in many aspects. SM does not only contribute to customer relationship management, but also support intra and inter-organizational issues such as employee engagement and partner collaboration. However, the benefits of SM can only be realized if the firms successfully engage with their stakeholders in SM network.

\subsection{Determinants of Stakeholder Engagement in Social Media}

Stakeholder engagement has gained increasing attention since the introduction of SM and its broad usage by firms (Viglia et al, 2018). SM accelerates the communication and enables the interaction and dialogue between the organizations and individuals. Consequently, organizations can promote their commercial, organizational, and CSR activities at a low cost to engage their stakeholders. For instance, Lee et al. (2013) found that social media offers favorable communication advantages to socially responsible firms.

In B2B context, individuals who have similar professional interests can easily be connected to each other through SM (Huotari et al., 2015). According to Huotari et al. (2015), two classes of B2B SM users exist: internal users who are either the corporate itself or internal and external users who consist of corporate users, customers of the corporate, related professional stakeholders, and individuals who are interested in the company but do not have any professional tie. These individuals are stakeholders of the company and can play a significant role in distributing and creating the SM content if the engagement is successful.

Stakeholder engagement, on the other hand, is challenging in SM. The posted SM content significantly affects the level of stakeholder engagement. That is, the number of likes, comments, and re-shares are heavily influenced by the characteristics of the post such as its media type, timing, content type, and length of message. Managers need to consider all aspects of SM thoroughly, such as whether to share the post with a picture or video, how many words to include in a message, or content type of the post. 
Although not many, some papers have attempted to find out the determinants of higher stakeholder engagement via SM. Based on our literature review and thorough investigation of the SM posts of the container lines, we have developed some propositions regarding what can influence the engagement rate of stakeholders. The elements of these propositions will be used as the independent variables in our CHAID analysis.

\section{Fluency of message}

McShane et al. (2019) argued that since users of SM are usually cognitively busy, they tend to like fluent posts, in which it is easier to process information. The fluency of a SM post is influenced by including a hashtag, tagging or mentioning another user, and length of the message. Inclusion of a hashtag or tagging another user are usually aimed to increase the engagement of stakeholders. Yet, in practice, the result may be just the opposite as hashtags and tags may cause lack of visual clarity and extra effort for translation of symbols (Pancer and Poole, 2016). In parallel to this argument, McShane et al. (2019) analyzed the SM engagement levels of the top 50 B2B companies' Twitter posts. They found that use of hashtags and difficulty in the text decreases the engagement (likes and shares) of the posts. Similarly, Dodson (2016) also suggests that the shorter the message, the greater the user engagement. Accordingly, we have the following proposition.

Proposition 1: Fluency of container lines' Tweets influences the engagement rate of their stakeholders

\section{Content type}

The content of container lines' SM post may be related to different subjects. A container line can share a new service as an advertisement, environmental initiatives as a part CSR, sectoral information, or celebration of a special day. The content of what container lines share may affect the engagement rate of followers. For instance, Denktas-Sakar and Surucu (2018) investigated the Facebook posts of the top 30 3PL logistics companies and found that content type of posts significantly influences the stakeholder engagement on SM. The authors indicated that there is a positive correlation between the ratio of sharing special day celebration posts and the engagement rate whereas there is a negative relation between the ratio of sharing industrial information and engagement rates. Cvijikj and Michahelles (2013) found that entertaining posts lead to higher levels of likes, comments, and shares compared to informative and remuneration contents. Khan et al. (2016) also ascertained that the engagement level may change based on whether the post is informative or entertaining.

Proposition 2: The content type of container lines' Tweets influences the engagement rate of their stakeholders

\section{Vividness level}

Like other SM platforms, Twitter allows its users to embed a media such as a photo, a gif, or a video. If the Tweet has no embedded media and only consists of texts, then it has a low vividness. If it includes a photo, then it has a medium vividness while it has high vividness if the embedded media is a video or a gif. 
Previous literature does not have an equivocal view on the relationship between vividness level or type of media and the engagement rate. For instance, McShane et al. (2019) found that tweets with an image or a video have higher engagement levels. Viglia et al. (2018) also found that Facebook posts with photos and videos cause higher number of likes. Denktas-Sakar and Surucu (2018) ascertained that Facebook posts with photos yield higher engagement level compared to videos and texts. Cvijikj and Michahelles (2013) found that photos and status posts generate higher engagement level than videos and links. Khan et al. (2016) found that highly vivid posts are the ones that drive the highest engagement rates in Facebook pages of fast food companies. Despite the conflicting results, the majority of the findings support that vividness or media type of a post affects the engagement level. Accordingly, our proposition is as below.

Proposition 3: The vividness level of container lines' Tweets influences the engagement rate of their stakeholders.

\section{Tangibility of resources}

As can be seen in the SM posts of container lines, they frequently share their resources on SM via an image or video. Some of these resources are tangibles such as a ship or a container while others are intangible ones such as people working in the company or the company's logo. Tangible and intangible resources are crucial to achieving and sustaining competitive advantage and firm performance (Greco et al., 2013; Kamasak, 2017). Tangible resources include financial or physical assets whereas intangible resources consist of non-physical assets such as intellectual property and brands. The literature indicates that tangible and intangible resources do not have the same level of contribution to a firm performance or success (Galbreath, 2005; Kamasak, 2017). We envisage that this discrepancy may also lead to a difference in the perception of stakeholders, and may affect their engagement level. Besides, tangibles are an important dimension of service quality (Parasuraman et al., 1985). Since services are intangible in nature, the presence of tangible assets can increase the perceived quality of customers. The tangible element plays an important role in the perception of service quality in the container shipping market too (Yuen and Thai., 2017). It was found that tangibility is a crucial service quality signaling tool in the website of motor carriers (Williams et al., 2018). Hence, we posit the below proposition.

Proposition 4: Tangibility of resources in container lines' Tweets influences the engagement rate of their stakeholders.

\section{Existence of a link}

In Twitter messages, it is possible to include an external link that users may click on and get deeper insights of a topic. Previous studies have examined whether the existence of a link affects engagement rate. Viglia et al. (2018) analyzed the SM posts of a closed Facebook group, EXPO 2015. The authors found that the inclusion of an external link has a significant positive impact on interactive engagement, number of comments in other words. In contrary, Pancer and Poole (2016) ascertained that an external link in the Tweet caused a lower level of engagement in politicians' election accounts. Despite the conflicting findings regarding the inclusion of an external link to garner engagement, their results indicate a relationship between the two. Accordingly, our proposition is provided below. 
Proposition 5: Existence of a link in container lines' Tweets influences the engagement rate of their stakeholders.

\section{Existence of a call-to-action}

Call-to-action (CTA) or level of interactivity is commonly used in marketing to encourage the followers to participate actively in a SM post (Escobar-Rodriguez and Bonson-Fernandez, 2017). Sentences such as "buy now", "call now", and "book now" are some examples of CTA. Earlier studies have investigated the relationship between CTA usage and stakeholder engagement in B2C context. Escobar-Rodriguez and Bonson-Fernandez (2017) analyzed the fashion retailers' Facebook posts. They found that more than half of the posts included CTA and recommended that other firms should also consider this trend. Similarly, Khan et al. (2016) examined the Facebook posts of five different brands. They ascertained that CTA positively impacts stakeholder engagement. To examine CTA usage in B2B context and examine whether CTA has an influence on stakeholder engagement rate, we posit the following proposition.

Proposition 6: The existence of a CTA in container lines' Tweets influence the engagement rate of their stakeholders.

\section{Methodology}

This paper utilizes CHAID analysis which employs several independent variables and a dependent variable to classify the posts. We explain the sampling, data collection, the variables used in the analysis, and the details of the CHAID method here.

\subsection{Sampling and Data Collection}

Twitter is the most commonly used micro-blogging site with 330 million monthly users (Zephoria, 2019; Alexa, 2018). Each day, some 500 million tweets are shared, which means that 5,787 posts are tweeted every second (Cooper, 2019). Twitter enables dual conversation between users (Kietzman et al., 2011). This SM platform provides firms to reach out to a vast range of stakeholders (Bonson et al., 2016) and engage with them (Lovejoy et al., 2012). By using Twitter, organizations can run marketing campaigns, advertising, and public relations activities (Stelzner, 2018). In fact, Twitter is one of the most commonly used SM platforms by B2B organizations (Brandwatch, 2015; Swani et al., 2014).

This study focuses on global container lines' Twitter pages. We chose the study's sample from the Alphaliner Top 100 list. We targeted the first five largest container lines as our targeted sampling frame. The chosen firms are APM-Maersk, MSC, COSCO, CMA CGM, and HapagLloyd. Although COSCO has a Twitter account, during the data collection period COSCO only posted two tweets. Thus, we excludedCOSCO. The remaining four container lines exceeded $50 \%$ of total TEU capacity of the world fleet at the time of data collection. As of February 1, 2019 Maersk had 75,041 followers, CMA CGM had 23,857 followers, Hapag-Lloyd had 8,550, followers, and MSC had 4,910 followers. We manually collected the Tweets of these four lines for the period between $1^{\text {st }}$ of September, 2018 and $31^{\text {st }}$ of January, 2019. The data collection was completed in February, 2019. A total of 488 tweets were collected.

\subsection{Data Coding}


Regarding the coding procedure, we adopted De Vries et al. (2012)'s and Luarn (2015)'s coding protocol. Two coders, who are authors of this paper, participated in the coding procedure. After receiving a 100 minutes training about the coding procedures, both coders coded four firms' posts shared between 1 September 2018 and 31 January 2019 separately. After the coding is completed, the coders compared their results, and when a dispute occurs about the coded posts, the coders re-analyzed disputed posts to reach a consensus. To calculate inter-coder reliability, we adopted Perreault and Leigh's (1989) method. According to their method, the inter-coder reliability should be between $0.8-1.0$ to obtain a reliable coding result. Our inter-coder reliability is 0.89 , which is acceptable.

For this research, we used eight independent variables and one dependent variable.

\section{Independent variables}

We used three independent variables to measure the fluency of messages: "Length of messages", "hashtag usage", and "mentioning another firm". "Length of message" is the only metric independent variable in the study. Twitter allows a maximum of 280 characters in tweets. We measured the length of a message as the number of characters used in a tweet message. "Hashtag usage", on the other hand, is a categorical independent variable. Coders coded each tweet whether they have any hashtags (Yes / No). Regarding "mention another firm", we had two categories, which are yes and no.

Content type consists of five categories: "company news", "sectoral information", "advertisement", "corporate social responsibility (CSR)", and "celebration". When an organization provides information about their firm, such as their financial performance, it was coded as company news. If a tweet gives information about the shipping industry, it was coded as sectoral information. For instance, MSC Cargo's tweet dated 12 October 2018 mentioned a need for the transparency of the cargo declaration (MSC Group, 2018). Tweets were coded as an advertisement if they promote the company's new or existing services. For instance, HapagLloyd's tweet dated 17 January 2019 promoted the Hapag-Lloyd's new tool named Quick Quotes which is used for price estimations (Hapag-Lloyd, 2019). If an organization celebrates a special day such as New Year, mother's day, fests, and holidays, it was coded as a celebration. Finally, there are also shares or tweets CSR. For example, Maersk's tweet dated 3 October 2018 shared their donation of Maersk to Hurricane Florence survivors (Maersk, 2018).

Vividness level consists of three categories which are "low", "medium", and "high". Low vividness means that tweet contains only sentences or links. There are no visual elements in the tweet. Medium vividness means that photo(s) are embedded in the tweet. Lastly, when a tweet includes video or gif, it is coded as high vividness.

Tangible assets are the physical assets that people can touch and feel such as buildings, ships, containers, port equipment, and etc. while intangible assets are the nonphysical assets that such as IT systems, know-how, trademarks, and relations etc. For container lines, it is a common practice to share photos and videos of both their tangible and intangible assets. For this independent variable, we had three categories which are tangible, intangible and non-applicable (N/A). So, if a container line shares a tweet which includes tangible assets, it was coded as tangible, and if a container line shares a tweet which includes intangible assets, it was coded as intangible. Lastly, since some of the tweets did not contain any photo or video, these tweets were coded as N/A by the coders. All of the 488 tweets were coded accordingly. 
Existence of a link has two categories which are yes and no. If an external link was embedded in the tweet, it was coded as yes. But if there was no link embedded in the tweet, it was coded as no. Existence of a CTA also consists of two categories. If a tweet includes sentences such as "try it now", "call us now", or "explore now", it was coded as yes. If a tweet does not include such sentences, then it was coded as no.

\section{The dependent variable}

The dependent variable in our CHAID analysis is the stakeholder engagement rate. To calculate the stakeholder engagement rate, we used the formula that are developed by Bonson and Ratkai (2013) and Bonson et al. (2016), which can be used for both Facebook and Twitter. As illustrated in Table 1, the number of interactions which are "likes", "comments", and "retweets" are required for computing the engagement rate. Thus, the coders collected all tweets' like, comment, and re-tweet numbers.

Table 1. Formulas used in measurement of stakeholder engagement rate

\begin{tabular}{|c|c|c|}
\hline Name & Formula & Measures \\
\hline \multicolumn{3}{|l|}{ Popularity } \\
\hline $\mathrm{P} 2$ & Total likes / total number of tweets & Average number of likes per tweet \\
\hline P3 & (P2 / number of followers) $\times 1,000$ & $\begin{array}{l}\text { Average number of likes tweet per } \\
1,000\end{array}$ \\
\hline \multicolumn{3}{|l|}{ Commitment } \\
\hline $\mathrm{C} 2$ & $\begin{array}{l}\text { Total comments / total number of } \\
\text { tweets }\end{array}$ & $\begin{array}{l}\text { Average number of comments per } \\
\text { tweet }\end{array}$ \\
\hline $\mathrm{C} 3$ & (C2 / number of followers) $x 1,000$ & $\begin{array}{l}\text { Average number of comments tweet } \\
\text { per } 1,000\end{array}$ \\
\hline \multicolumn{3}{|r|}{ Pu 1,000 - 5} \\
\hline $\mathrm{V} 2$ & Total re-tweets / total number of tweets & $\begin{array}{l}\text { Average number of re-tweets per } \\
\text { tweet }\end{array}$ \\
\hline V3 & (V2 / number of followers) $\times 1,000$ & $\begin{array}{l}\text { Average number of re-tweets tweet } \\
\text { per } 1,000\end{array}$ \\
\hline
\end{tabular}

Resource: adopted from Bonson and Ratkai, 2013; Bonson et al., 2016.

To be able to calculate the stakeholder engagement rate, we followed the procedure of Bonson and Ratkai (2013) and Bonson et al. (2016). After gathering the number of likes, comments, and re-tweets, we calculated the average number of likes per tweet (P2), the average number of comments per tweet (C2) and the average number of re-tweets per tweet (V2), respectively. $P 2$ was calculated by dividing the total likes to total number of tweets. The same calculation was made for comments (C2) and re-tweets (V2). After calculating $P 2, C 2$ and V2, we calculated average number of likes tweet per 1,000 (P3), average number of comments tweet per 1,000 (C3) and average number of re-tweets tweet per 1,000 (V3). P3 was calculated by diving the $P 2$ to the number of followers and multiplying the obtained value with 1,000 . Dividing average number of likes to number of followers help mitigate the possibility that engagement levels of tweets may be affected by the difference in container lines' number of followers. The same calculation was made for comments (C3) and re-tweets (V3). Finally, the stakeholder engagement rate was achieved by summing up the $P 3, C 3$ and $V 3$ values. 


\subsection{CHAID Method}

This paper aims to classify SM posts of container lines based on their engagement rates. This will help researchers to understand the types of post leading to a greater engagement level. We conduct a Chi-Square Automatic Interaction Detection (CHAID). It is a decision tree methodology used for classification and prediction. Decision trees, which are data-mining tools, implement thorough statistical analysis but present visually easy results to aid interpretation. CHAID builds non-binary trees by splitting the whole dataset into homogenous subgroups based on the interaction between predictor variables and the dependent variable (Kass, 1980). In other words, CHAID algorithm splits the sample into exhaustive sub-groups that differ based on the dependent variable. In addition, CHAID is a non-parametric technique that does not require the assumption of normal distribution (Díaz-Pérez and Bethencourt-Cejas, 2016).

In CHAID analysis, there is one dependent (criterion) variable and multiple independent (predicting) variables. Both dependent and independent variables can be either metric or nominal (Waara et al., 2016). CHAID consists of several steps and at each step, the CHAID algorithm compares the predictor variables and determines the best predictor for partitioning. First, the most significant predictor partitions the whole dataset and then the partitioned data is further split by the next most significant predictor variable, which creates nodes (Díaz-Pérez and Bethencourt-Cejas, 2016). The node formation process continues until no significant relation exists between an independent and the dependent variable. That is, the process continues until no heterogeneous node exists with respect to the interaction between the dependent and independent variables.

If the dependent variable is continuous, CHAID algorithm utilizes F tests for partitioning, and if the dependent variable is nominal, then it uses chi-square statistic (Ozgulbas and Koyuncugil, 2006). Based on conventions, we set the significance level to be 0.05 for splitting the nodes. The first node is called a root node, which appears on top and includes the entire sample. The partitioned nodes are named parent nodes if they are split into subgroups. If no more split occurs, then that group is called child node. We adjusted the parent/child node ratio as 40/20 since we have a relatively small sample for a decision tree analysis (McCormick et al., 2017). It means the smallest number of a parent node can be a minimum 40 whereas the smallest number of a child node can be minimum 20. We have adjusted the tree growth limit to a maximum of five, which means that splitting stops when the tree branches out to a maximum five layers. We used SPSS version 21 to conduct the CHAID analysis.

Decision tree methodology is used in several disciplines such as tourism (Díaz-Pérez and Bethencourt-Cejas, 2016), travelling (Xiong and Zhang, 2014), and public transportation (Waara et al., 2016). Although various studies in different sectorial areas have conducted CHAID methodology with the purposes of segmentation, prediction, and classification, the methodology is not common in freight transportation area. Regarding SM studies in the literature, no study has used CHAID to determine the type of post groups that result in greater engagement.

4. Results 


\subsection{Descriptive Results}

The descriptive results illustrate that only $14 \%$ of the posts are re-tweets while $86 \%$ are the posts originally created by the container lines. As shown in Table 2, around $46 \%$ of the posts have an external link that stakeholders can click and get more detailed information about a content. Approximately $70 \%$ of the tweets include one or more hashtags while almost $30 \%$ do not have any. $21 \%$ of the tweets, on the other hand, include a CTA. Around $60 \%$ of the posts mention another company such as a supplier, customer, or partner.

Table 2 Descriptive statistics of binary dependent variables

\begin{tabular}{lllllllll}
\hline & \multicolumn{2}{c}{ Existence of a link } & $\begin{array}{l}\text { Existence } \\
\text { hashtag }\end{array}$ & of a & Existence of CTA & \multicolumn{2}{c}{$\begin{array}{l}\text { Mention of another } \\
\text { firm }\end{array}$} & \\
\hline & Number & Percent & Number & Percent & Number & Percent & Number & Percent \\
\hline Yes & 226 & $46 \%$ & 369 & $71 \%$ & 103 & $21 \%$ & 297 & $61 \%$ \\
No & 262 & $54 \%$ & 139 & $29 \%$ & 385 & $79 \%$ & 191 & $39 \%$ \\
Total & 488 & $100 \%$ & 488 & $100 \%$ & 488 & $100 \%$ & 488 & $100 \%$ \\
\hline
\end{tabular}

As shown in Table 3, only $8 \%$ of the posts have high vividness (videos and gifs) whereas a great majority (60\%) of the posts have medium vividness (photos) and $23 \%$ have low vividness. Around $36 \%$ of the posts involve a tangible resource element such as a ship or container while almost half of the posts have intangible resources and $17 \%$ do not have any resource element. Among the videos and images, a large majority of the posts are real videos or images while a small amount of the posts are graphics created or drawn by the account holder.

Table 3 Descriptive statistics of variables with three categories

\begin{tabular}{llllll}
\hline Vividness & \multicolumn{3}{c}{ Tangibility of resources } \\
\cline { 2 - 3 } \cline { 5 - 6 } & Number & Percent & & Number & Percent \\
\hline High & 38 & $8 \%$ & Tangible & 178 & $36 \%$ \\
Medium & 336 & $69 \%$ & Intangible & 229 & $47 \%$ \\
Low & 114 & $23 \%$ & No Resource & 81 & $17 \%$ \\
Total & 488 & 100 & Total & 488 & 100 \\
\hline
\end{tabular}

As shown in Table 4, regarding the content type of the posts, the largest proportion belongs to advertisement category with over $37 \%$ followed by company news with over $27 \%$. CSR is the third largest category followed by sectoral information, and celebration posts. Number of characters is the only metric predictor variable and the mean of all Tweets in our sample is 190 characters.

Table 4 Descriptive results of content type categories

\begin{tabular}{lll}
\hline Content & Number & Percent \\
\hline Celebration & 13 & $2.7 \%$ \\
CSR & 112 & $23 \%$ \\
\hline
\end{tabular}




\begin{tabular}{lll}
\hline Company News & 133 & $27.3 \%$ \\
Advertisement & 182 & $37.3 \%$ \\
Sectoral Information & 48 & $9.8 \%$ \\
Total & 488 & $100 \%$ \\
\hline
\end{tabular}

\subsection{CHAID Decision Tree Results}

As shown in Figure 2 and 3, the results indicate that there are a total of 19 nodes consisting of 1 root node, 8 parent nodes, and 10 child nodes. The largest parent node (Node 1) has 343 posts while the smallest parent node (Node 12) has 71 members. The largest childe nod (Node 5) has 116 posts while the smallest child node (Node 10) has 24 SM posts.

According to the results, the mean value of engagement level is 2.30 for the entire 488 tweets. Eight of the variables partition the dataset significantly: number of characters, tangibility of resources, use of hashtag, existence of a link, content type, vividness of the post, existence of a CTA, and mention of another company. The CHAID results indicate that the first significant predictor is the number of characters. As shown in Figure 2, Tweets that have 225 or lower characters, Node 1, have significantly higher engagement level (mean=2.73) compared to those with longer Tweets, Node 2 (mean=1.29). The posts in Node 2 are separated into two by the use of hashtag. Accordingly, the posts without a hashtag (Node 6) generate a higher engagement level (mean=2.14) than the posts including one or more hashtags (mean=1.09).

The posts with 225 and lower characters are split into two by tangibility of resources. Posts that feature tangible resources (Node 4) lead to greater engagement level (mean=3.62) compared to those in Node 3 which feature intangible resources or without resource elements (mean=2.15). As illustrated in Figure 3, the existence of a link partitions Node 3. The posts with a link (Node 7) have an engagement mean of 2.56 while the posts without a link (Node 8 ) have a mean value of 1.72. Vividness significantly splits Node 8 into two sub-groups: High vividness (Node 11) and Medium and Low Vividness (Node 12). Tweets with high vividness, videos or gifs, have significantly higher engagement level (mean=2.53) than posts with medium and low vividness (mean=1.37). Under the sub-group of medium and low vividness, posts with 192 or lower characters generate a higher engagement rate (mean=1.83) than those with above 192 characters (0.82).

On the other hand, Node 4, the posts with 225 or lower characters and with tangible resources, are partitioned into two by content type. The Tweets which are about CSR, special day celebration, or combination of different contents (Node 9) have a greater engagement level than those which are about advertisement, sectoral info, or company news (Node 10). Node 9 is also partitioned into two as Node 13 and Node 14 by the existence of a CTA. Posts with CTA causes a greater engagement score (mean=3.97) than without CTA posts (mean=2.51). Node 13, which consists of posts without a CTA, is split by whether a post mentions another firm. The posts that do not mention another firm in the message have a higher score (mean=3.11) than those that mention (mean=2.11). 


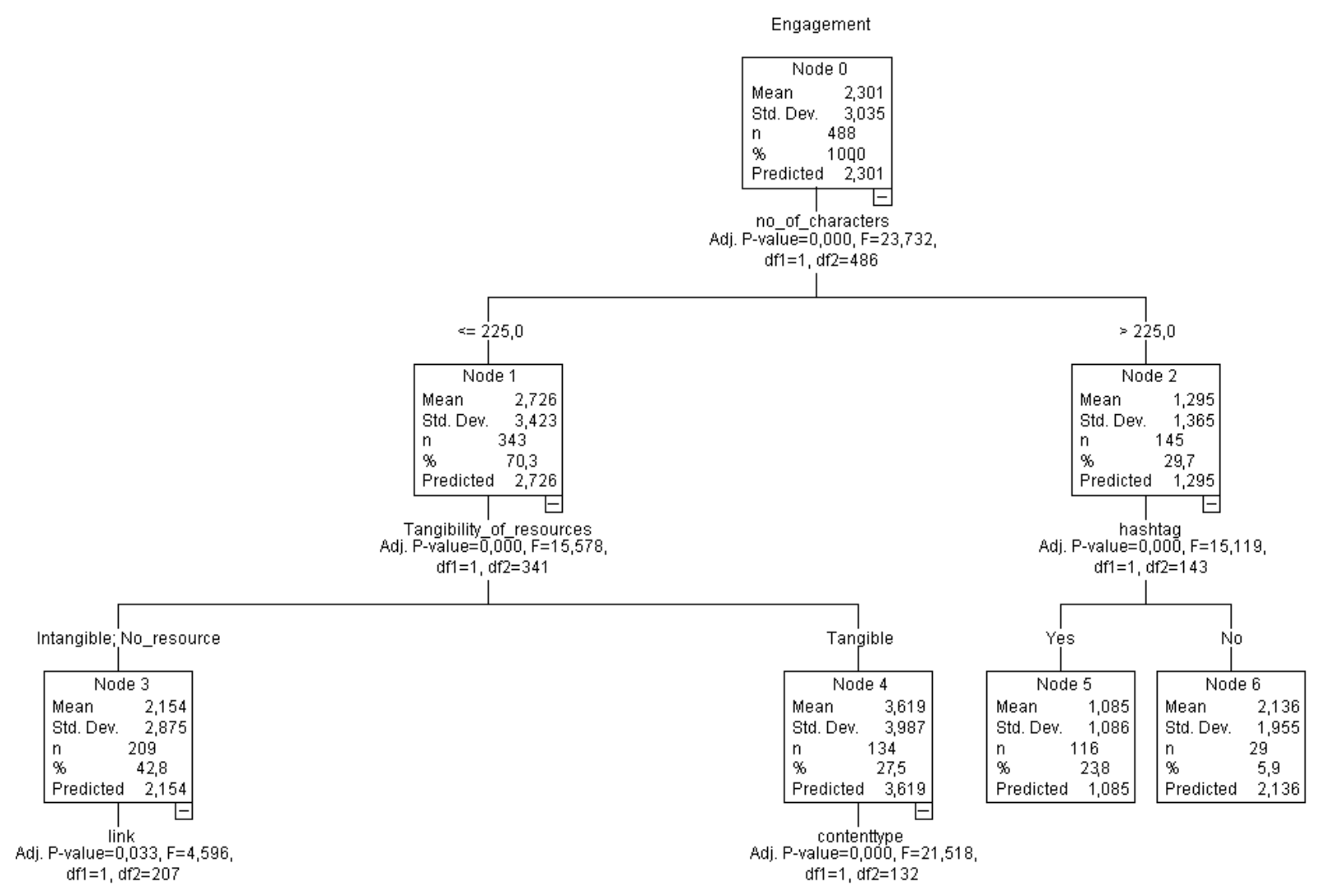

Figure 2 CHAID Decision Tree Display (Part 1*)

*The decision tree is displayed in two separate figures as Part1 and Part2 due to its large size 


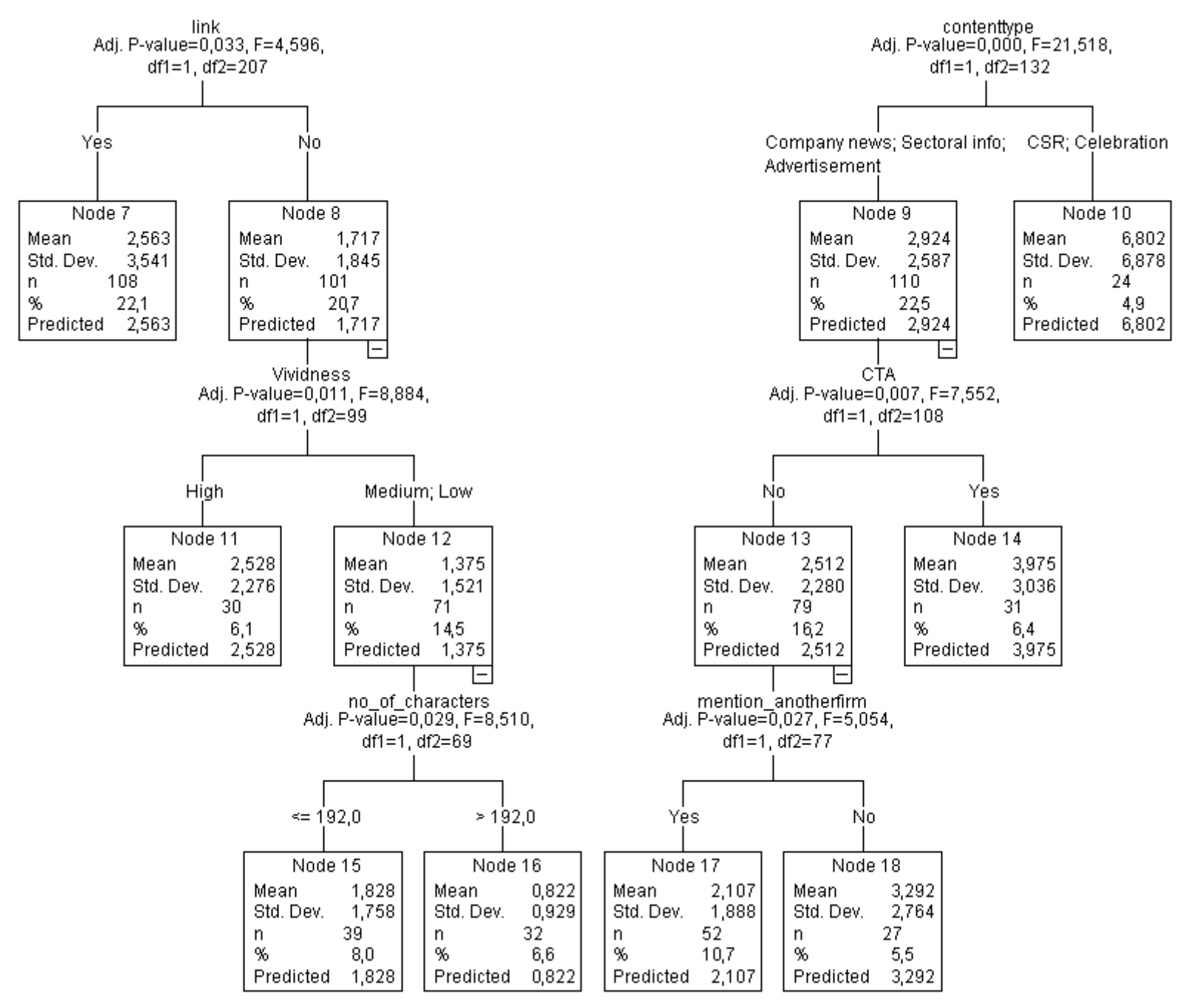

Figure 3 CHAID Decision Tree Display (Part 2)

According to the results, the most engaging group of posts is Node 10, which have 225 or lower characters, feature tangible resource elements, and content of CSR and celebration. Node 14 is the second most engaging sub-group which resembles Node 10 in terms of length of message and tangibility of resources. However, it differs with respect to the content type, which includes the contents of company news, sectoral info, and advertisement. Further, it also consists of posts with a call-to-action. The third most engaging sub-group is Node 18, which is similar to Node 14 except tweets in this sub-group do not have a CTA and do not mention another firms at all.

Table 5. The descriptive of child notes

\begin{tabular}{llll}
\hline Child Node & Population & Percentage & Engagement Mean \\
\hline Node 10 & 24 & $4.9 \%$ & 6.802 \\
Node 14 & 31 & $6.4 \%$ & 3.975 \\
Node 18 & 27 & $5.5 \%$ & 3.292 \\
Node 7 & 108 & $22.1 \%$ & 2.563 \\
Node 11 & 30 & $6.1 \%$ & 2.528 \\
Node 6 & 29 & $5.9 \%$ & 2.136 \\
\hline
\end{tabular}




\begin{tabular}{llll}
\hline Node 17 & 52 & $10.7 \%$ & 2.107 \\
Node 15 & 39 & $8.0 \%$ & 1.828 \\
Node 5 & 116 & $23.8 \%$ & 1.085 \\
Node 16 & 32 & $6.6 \%$ & 0.822 \\
Total & 488 & $100 \%$ & \\
\hline
\end{tabular}

Regarding the least engaging subgroups, or child node in other words, Node 16 is the least engaging one which includes posts with a number of characters between 192 and 225, with no external link, and with medium or low vividness. The second least engaging subgroup is Node 5 which consists of posts with over 225 characters and include at least one hashtag.

\section{Discussion}

The results of this study demonstrate that success of stakeholder engagement via SM is affected by the various characteristics of the shared posts. Our findings indicate that the independent variables utilized in the analysis successfully partitioned the total sample in relation with the engagement rate. The results approved all of the propositions developed in our literature review. Regarding fluency of the message, the use of hashtags, length of message, and mentioning another company in the message influence the engagement rate of stakeholders. CHAID output illustrates that the Tweets have a lower engagement rate if the message is lengthy, has one or more hashtags, and mentions another company. In other words, similar to the findings of McShane (2019) and Pancer and Pooler (2016), more fluent messages yield greater engagement rate in our sample. The very low engagement rate of Node 5 - which consists of posts with 225 and more characters and with hashtags - also suggests that influent messages cause lower engagement rates.

In fact, inserting hashtags is actually aimed to reach a wider audience and attract the attention of more users because the Tweets are displayed to the users who search for a specific hashtag such as \#climatechange, \#economicgrowth, or \#southafrica. However, those users are not necessarily the stakeholders of the company. The inclusion of a hashtag certainly enhances the visibility of the line in SM, but it does not guarantee an increase in the engagement rate. Besides, as McShane (2019) suggested, hashtags increase the difficulty of text and lead to a lower level of engagement. Similarly, tagging another company or a user also complicates the fluency. Moreover, mentioning another company may distract or alienate the stakeholders who are simply not interested in the mentioned company. Thus, when a container line tags another company such as a customer or a supplier, the stakeholders, who follow the line's Twitter account, may feel reluctant to be engaged with the Tweet.

The results indicate that stakeholders engage more to a container line's Tweet with tangible resources compared to the ones with intangible resources or no resource. This finding is consistent with the service quality literature which suggests the utilization of tangible elements to increase perceived quality. The tangible resources such as a vessel or building are concrete elements that may improve stakeholders' perception of a positive image regarding the 
reputation of the company. This result concurs with the study of Williams et al. (2018). The authors consider using tangibles as an effective signal of service quality for carriers' websites.

The content type of the Tweet also significantly partitioned the posts that have lower number of characters and tangible resources. In particular, CSR and celebration posts boost the engagement rate more than business related posts such as advertisement or industry news. This result is consistent with findings of Denktas-Sakar and Surucu (2018) who also found that celebration posts increase the engagement rate of 3PL companies' stakeholders. Our results also support the findings of Lee et al. (2013) who stated that socially responsible firms benefit more from user-driven communication. Lee et al. (2013) found that firms having higher CSR ratings build a greater presence in social media and build faster. The higher engagement level of CSR posts in our study indicates a partial support for their findings as well.

The fact that celebration and CSR posts receive more engagement is quite logical because the social posts are more joyous and emotional in nature. For instance, a post celebrating the new year or mentioning the container line's initiative on global warming can attract a larger number of stakeholders. Hence, they can garner more interest from stakeholders. On the contrary, for instance, an industry news post which discusses the effect of blockchain in shipping industry can only attract those stakeholders interested in the topic. Besides, in accordance with the findings of Cvijikj and Michahelles (2013), the availability of Celebration and CSR posts may possess more entertaining contents which can explain why they yield higher engagement rates.

Among the posts without a tangible resource, those with an external link generate a higher stakeholder engagement. Khan et al. (2016) suggested that informative posts may cause a greater engagement rate. Since external links enable users to obtain deeper insights into a topic or access richer contents, it is not surprising that existence of a link generate more engagement. As Lovejoy et al. (2012) also suggest use of links may help companies overcome word limit of Twitter if more information is needed to convey. Besides, some of the links also direct stakeholders to the websites of container lines where a rich amount of tangible resources and various interesting contents exist. This may explain why external links are effective when Tweets do not have any tangible resource.

The results showed that vivid posts, those with a video or a gif, cause a higher engagement rate compared to medium (photos) and low vivid (texts) ones among the posts with less characters, no tangible resource as well as external link. The result is logical because videos or gifs are usually more entertaining. They can also have more informative content compared to pictures or only texts. In the event when there is no external link that stakeholders retrieve detailed information about a topic, videos and gifs can serve as substitutes to help stakeholders obtain more information, which can explain why they cause higher engagement.

6. Theoretical and practical implications

This paper contributes to literature in several aspects. First, this study contributes to the stakeholder engagement by investigating SM as a channel for stakeholder engagement. Stakeholder engagement proposes the involvement of stakeholders in dialogue to determine their social, environmental and economic concerns about the firm to improve its decisionmaking and accountability (Herremans et. al., 2016). Stakeholder engagement involves a series of process which includes stakeholder map, engagement model, stakeholder concerns to action 
plan. This paper contributes to the segment on engagement model. In particular, it determines how SM contents should be structured and designed to improvement stakeholder engagement.

Our paper also extends the knowledge on stakeholder engagement literature in a B2B services industry, container shipping. The stakeholders of container shipping are diverse due to the environmental and commercial complexity and internationality of the business. The SM offers numerous opportunities for container lines to enhance commitment of their stakeholders in such a complicated environment. However, the literature in container shipping has not investigated how SM posts should be managed at the tactical or operational level. Our paper fills this gap by determining the SM post characteristics that influence the engagement rate. We expect our paper to motivate scholars to conduct studies on the SM strategies of container shipping and other freight transporters. This is essential considering the potential benefits and power of SM.

To the best knowledge of authors, this study is the first that finds out SM post classes based on the interaction between their characteristics and engagement rates by employing a decision tree methodology. The previous studies in our literature review found the determinants of SM engagement rates by conducting aggregated analysis to SM posts and drew generic conclusions. However, our study investigates the subgroups of SM posts instead of examining all SM posts together and drawing generic conclusions. This enables more refined results and help both researchers and practitioners to have a finer perception on the SM behavior of stakeholders.

Our study is also one of the few articles that examine SM in freight transportation area (Buratti et al., 2018) and apply a decision tree analysis in freight transportation. CHAID, a decision tree method, has been used in behavioral researches in travelling and public transportation (Waara et al., 2015), but it has not been utilized in freight transportation. Our study can motivate freight transportation researchers, specifically in the shipping domain to apply a decision tree method for classification and prediction. This is necessary considering the heterogeneous characteristics and expectations of freight shippers.

Another significant contribution of our study is that we utilized a large variety of determinants of stakeholder engagement in SM. We found that a total of eight determinants of SM posts significantly influence the engagement rate in the sample. Moreover, to the best of our knowledge, tangibility of resources has been used for the first time as a determinant of engagement. The utilization of a large number of determinants and their significant influence on engagement can encourage future studies to consider more dimensions of SM posts when investigating stakeholder engagement rates.

Our paper also extends the knowledge of SM in B2B markets. For instance, call-to-action is mostly studied in consumer researches as a determinant of stakeholder engagement. However, the sampled container lines also employ CTA in their posts in the purpose of engagement. We tested if employment of CTA has any effect on the engagement level and found that existence of a CTA significantly splits a segment of our sampled SM posts. Our findings show that CTA posts have a greater engagement level in the container shipping market.

The paper also offers managerial implications for container lines as well as other B2B services companies. Our literature review clearly indicates how SM can bring numerous advantages to B2B companies (Jussila et al., 2014: Lam et al., 2016). These benefits can only be achieved if the companies successfully engage their stakeholders. At this point, it is vital for container line managers to effectively manage their SM posts. Our paper helps managers understand what 
kind of SM posts lead to a higher stakeholder engagement. By this way, they can utilize SM as a more effective channel of stakeholder engagement.

Our results demonstrate that the highest engaging SM post group comprised of posts with less than 225 characters, with tangible resources, and with more entertaining and social contents such as CSR or special day celebrations. Thus, we suggest managers to keep their posts short, include a tangible resource such as a ship if possible, and share more social and entertaining posts. If sharing tangible resources is not possible, then we suggest them to include an external link. Managers should also share high vivid posts such as a video or a gif especially when a tangible resource or an external link is lacking. Managers can also share more interactive posts including a CTA to increase the engagement rate.

We acknowledge that SM managers of companies should diversify their messages to avoid repetition. This means that they cannot only focus on highly engaging posts. However, they can increase the ratio of these highly engaging posts. For instance, we have detected that the ratio of videos and gifs is quite low compared to photos and texts. Thus, it is possible that container line managers share more videos and increase the ratio of high vivid posts.

According to our results, we also suggest managers to avoid using lengthy messages, including hashtags, and tagging another company, which may deteriorate the fluency of the message and eventually decrease the engagement rate.

\section{Conclusion, Limitations, and Future Research}

This paper investigated Twitters of container lines as a channel of stakeholder engagement and found out the SM post characteristics that generate a higher stakeholder engagement. The CHAID analysis demonstrated that a total of eight characteristics of tweets influence the stakeholder engagement rate. The characteristics are number of characters, existence of a hashtag, tangibility of resources in tweet, existence of a link, content type, vividness, existence of a CTA, and mentioning another firm.

Our findings should be carefully generalized to other industries as the results may change based on the industry type. For instance, in our sample, tangible resources lead to higher engagement. One of the reasons might be because the sampled companies are B2B services companies, and stakeholders of services companies may attach more importance to tangible resources compared to product companies. This assumption requires a further investigation.

One of the limitations of this paper is that it does not identify or segment the stakeholders. Identifying the stakeholders and then segmenting them are the first two, and significant, steps of the stakeholder engagement process. The results would have been much finer if we identified the stakeholders in our sampled SM accounts. However, such information about the occupations and affiliations of the followers is not available due to privacy reasons.

In our research, although the selected timeframe included Christmas Holiday season, we noticed that companies do not share posts in the holiday season. There was only one post shared in total during Christmas season (Post date is $28^{\text {th }}$ of December, 2018). There might be a possibility 
that engagement levels may vary based on whether it is holiday season or not, but since only one post was shared in holiday season, our paper is not able to find this out.

The results of this paper are limited to a single SM platform. Our literature review on the determinants of stakeholder engagement shows that there are some misalignments between the findings of papers. This misalignment may arise because some of these papers investigate different SM platforms. Manetti et al. (2017), for instance, stated that interaction level in Facebook was higher than Twitter in public transportation SM account. Thus, a future study that examines two different SM platforms of the same companies and compare if any difference exists between the different SM platforms.

The quality of the media is also a matter of question that needs to be examined. We envisage that the quality of a picture and video can also affect the engagement rate. Future studies can also investigate this issue. More detailed analysis can also be conducted. For instance, it would be interesting if future studies investigate what kind of tangible resources or what kind of industry news attract more attention.

\section{References}

Agnihotri, R., Dingus, R., Hu, M. Y., \& Krush, M. T. (2016). Social media: Influencing customer satisfaction in B2B sales. Industrial Marketing Management, 53, 172-180.

Alexa. https://www.alexa.com/topsites/category/Computers/Internet/On_the_Web/Online_Communit ies/Social_Networking (Accessed date: 21.05.2019)

Ammirato, S., Felicetti, A. M., Della Gala, M., Aramo-Immonen, H., Jussila, J. J., \& Kärkkäinen, H. (2018). The use of social media for knowledge acquisition and dissemination in B2B companies: an empirical study of Finnish technology industries. Knowledge Management Research \& Practice, 1-18.

Balci, G. \& Cetin, I. (2017). Market segmentation in container shipping service: a qualitative study. Management Research Review, 40(10), 1100-1116.

Bernard, M. (2016). The impact of social media on the B2B CMO. Journal of Business \& Industrial Marketing, 31(8), 955-960.

Bonsón, E., \& Ratkai, M. (2013). A set of metrics to assess stakeholder engagement and social legitimacy on a corporate Facebook page. Online Information Review, 37(5), 787-803.

Bonsón, E., Bednárová, M., \& Wei, S. (2016). Corporate Twitter use and stakeholder engagement: An empirical analysis of the Spanish hotel industry. European Journal of Tourism Research, 13, 69-83.

Brandwatch (2015). B2B Social Media Report. Accessed from https://www.brandwatch.com/reports/b2b-social-mediareport/ (Accessed date: 20.05.2019) 
Brennan, R., \& Croft, R. (2012). The use of social media in B2B marketing and branding: An exploratory study. Journal of Customer Behaviour, 11(2), 101-115.

Broekemier, G., Chau, N. N., \& Seshadri, S. (2015). Social media practices among small business-to-business enterprises. Small Business Institute Journal, 11(1).

Buratti, N., Parola, F., \& Satta, G. (2018). Insights on the adoption of social media marketing in B2B services. The TQM Journal, 30(5), 490-529.

Cawsey, T., \& Rowley, J. (2016). Social media brand building strategies in B2B companies. Marketing Intelligence \& Planning, 34(6), 754-776.

Cheng, C. C., \& Krumwiede, D. (2018). Enhancing the performance of supplier involvement in new product development: the enabling roles of social media and firm capabilities. Supply Chain Management: An International Journal, 23(3), 171-187.

Cooper, P. (January 16, 2019). 28 twitter statistics all marketers need to know in 2019. https://blog.hootsuite.com/twitter-statistics/ (Accessed date: 21.05.2019)

Cvijikj, I. P., \& Michahelles, F. (2013). Online engagement factors on Facebook brand pages. Social Network Analysis and Mining, 3(4), 843-861.

Iberdrola, 2019. Engagement model with stakeholders: We make every effort to build solid relations that are based of trust.

De Vries, L., Gensler, S., \& Leeflang, P. S. (2012). Popularity of brand posts on brand fan pages: An investigation of the effects of social media marketing. Journal of Interactive Marketing, 26(2), 83-91

Denktas-Sakar, G., \& Karatas-Cetin, C. (2012). Port sustainability and stakeholder management in supply chains: A framework on resource dependence theory. The Asian Journal of Shipping and Logistics, 28(3), 301-319.

Denktaş-Şakar, G., \& Sürücü, E. (2018). Stakeholder engagement via social media: an analysis of third-party logistics companies. The Service Industries Journal, 1-24.

Díaz-Pérez, F. M., \& Bethencourt-Cejas, M. (2016). CHAID algorithm as an appropriate analytical method for tourism market segmentation. Journal of Destination Marketing \& Management, 5(3), 275-282.

Dodson, I. (2016). The Art of Digital Marketing: The Definitive Guide to Creating Strategic, Targeted, and Measurable Online Campaigns. John Wiley \& Sons.

Dreher, S. (2014). Social media and the world of work: A strategic approach to employees' participation in social media. Corporate Communications: An International Journal, 19(4), 344-356.

Erdoğmuş, İ. E., \& Cicek, M. (2012). The impact of social media marketing on brand loyalty. Procedia-Social and Behavioral Sciences, 58, 1353-1360. 
Escobar-Rodriguez, T. and Bonson-Fernandez, R. (2017). Facebook practices for business communication among fashion retailers. Journal of Fashion Marketing and Management: An International Journal, 21: 33-50.

Faci, N., Maamar, Z., Burégio, V., Ugljanin, E., \& Benslimane, D. (2017). Web 2.0 applications in the workplace: How to ensure their proper use?. Computers in Industry, 88, 1-11.

Fisher, R., McPhail, R., You, E., \& Ash, M. (2014). Using social media to recruit global supply chain managers. International Journal of Physical Distribution \& Logistics Management, 44(8/9), 635-645.

Freeman, R. E. (1984). Strategic management: A stakeholder approach. Boston, MA: Pitman.

Freeman, R. E., Wicks, A. C., \& Parmar, B. (2004). Stakeholder theory and "the corporate objective revisited". Organization science, 15(3), 364-369.

Foltean, F. S., Trif, S. M., \& Tuleu, D. L. (2018). Customer relationship management capabilities and social media technology use: Consequences on firm performance. Journal of Business Research.

Galbreath, J. (2005). Which resources matter the most to firm success? An exploratory study of resource-based theory. Technovation, 25(9), 979-987.

Gómez, M., Lopez, C., \& Molina, A. (2019). An integrated model of social media brand engagement. Computers in Human Behavior, 96, 196-206.

Greco, M., Cricelli, L., \& Grimaldi, M. (2013). A strategic management framework of tangible and intangible assets. European Management Journal, 31(1), 55-66.

Hapag-Lloyd. (2019). https://twitter.com/HapagLloydAG/status/1085897582222536705 (accessed date: 10.02.2019)

Heller Baird, C., \& Parasnis, G. (2011). From social media to social customer relationship management. Strategy \& Leadership, 39(5), 30-37.

Herremans, I. M., Nazari, J. A., \& Mahmoudian, F. (2016). Stakeholder relationships, engagement, and sustainability reporting. Journal of Business Ethics, 138(3), 417-435.

Huotari, L., Ulkuniemi, P., Saraniemi, S., \& Mäläskä, M. (2015). Analysis of content creation in social media by B2B companies. Journal of Business \& Industrial Marketing, 30(6), 761770 .

Iankova, S., Davies, I., Archer-Brown, C., Marder, B., \& Yau, A. (2018). A comparison of social media marketing between B2B, B2C and mixed business models. Industrial Marketing Management.

Jussila, J. J., Kärkkäinen, H., \& Aramo-Immonen, H. (2014). Social media utilization in business-to-business relationships of technology industry firms. Computers in Human Behavior, 30, 606-613. 
Kamasak, R. (2017). The contribution of tangible and intangible resources, and capabilities to a firm's profitability and market performance. European Journal of Management and Business Economics, 26(2), 252-275.

Kass, G. V. (1980). An exploratory technique for investigating large quantities of categorical data. Journal of the Royal Statistical Society: Series C (Applied Statistics), 29(2), 119-127.

Katona, Z., \& Sarvary, M. (2014). Maersk Line: B2B Social Media- "It's Communication, Not Marketing". California management review, 56(3), 142-156.

Khan, I., Dongping, H., \& Wahab, A. (2016). Does culture matter in effectiveness of social media marketing strategy? An investigation of brand fan pages. Aslib Journal of Information Management, 68(6), 694-715.

Kietzmann, J. H., K. Hermkens, I.P. McCarthy, and B.S. Silverstre (2011) Social Media? Get Serious! Understanding the Functional Building Blocks of Social Media. Business Horizons,54, 241-251

Kontsevaia, D. B., \& Berger, P. D. (2017). Analyzing Factors Affecting the Success of Social Media Posts for B2b Networks: A Fractional-Factorial Design Approach. International Journal of Business, Economics and Management, 4(6), 112-123.

Kuznetsov, A., Dinwoodie, J., Gibbs, D., Sansom, M., \& Knowles, H. (2015). Towards a sustainability management system for smaller ports. Marine Policy, 54, 59-68.

Lam, H. K., Yeung, A. C., \& Cheng, T. E. (2016). The impact of firms' social media initiatives on operational efficiency and innovativeness. Journal of Operations Management, 47, 28-43.

Lee, K., Oh, W. Y., \& Kim, N. (2013). Social media for socially responsible firms: Analysis of Fortune 500's Twitter profiles and their CSR/CSIR ratings. Journal of business ethics, 118(4), 791-806.

Lovejoy, K., Waters, R. D., \& Saxton, G. D. (2012). Engaging stakeholders through Twitter: How nonprofit organizations are getting more out of 140 characters or less. Public Relations Review, 38(2), 313-318.

Luarn, P., Lin, Y. F., \& Chiu, Y. P. (2015). Influence of Facebook brand-page posts on online engagement. Online Information Review, 39(4), 505-519

Maersk. (2018). https://twitter.com/Maersk/status/1062612683445469186 (Accessed date: 04.02.2019)

Maersk. (2018). https://twitter.com/Maersk/status/1047428343057010688 (Accessed date: 04.02.2019)

Manetti, G., Bellucci, M., \& Bagnoli, L. (2017). Stakeholder engagement and public information through social media: a study of Canadian and American public transportation agencies. The American Review of Public Administration, 47(8), 991-1009.

McCormick, K., Salcedo, J., Peck, J., \& Wheeler, A. (2017). SPSS Statistics for data analysis and visualization. John Wiley \& Sons. 
McShane, L., Pancer, E., \& Poole, M. (2019). The Influence of B to B Social Media Message Features on Brand Engagement: A Fluency Perspective. Journal of Business-to-Business Marketing, 26(1), 1-18.

Michaelidou, N., Siamagka, N. T., \& Christodoulides, G. (2011). Usage, barriers and measurement of social media marketing: An exploratory investigation of small and medium B2B brands. Industrial Marketing Management, 40(7), 1153-1159.

MSC Cargo. (2018). https://twitter.com/MSCCargo/status/1050711514989174785 (accessed date: 13.02.2019)

Ozgulbas, N., \& Serhan Koyuncugil, A. (2006). Application of Data Mining Method for Financial Profiling. Social Responsibility Journal, 2(3/4), 328-334.

Pancer, E., \& Poole, M. (2016). The popularity and virality of political social media: hashtags, mentions, and links predict likes and retweets of 2016 US presidential nominees' tweets. Social Influence, 11(4), 259-270.

Park, S. O., Na, H. J., \& Kwon, O. (2016). Comparative effect of company-driven SNS activity vs. consumer-driven SNS activity on firm value: Evidence from Facebook. Computers in Industry, 82, 186-195.

Parasuraman, A., Zeithaml, V. A., \& Berry, L. L. (1985). A conceptual model of service quality and its implications for future research. Journal of Marketing, 49(4), 41-50.

Pitt, C. S., Plangger, K. A., Botha, E., Kietzmann, J., \& Pitt, L. (2017). How employees engage with B2B brands on social media: Word choice and verbal tone. Industrial Marketing Management.

Quinton, S., \& Wilson, D. (2016). Tensions and ties in social media networks: Towards a model of understanding business relationship development and business performance enhancement through the use of LinkedIn. Industrial Marketing Management, 54, 15-24.

Rodriguez, M., Peterson, R. M., \& Krishnan, V. (2012). Social media's influence on businessto-business sales performance. Journal of Personal Selling \& Sales Management, 32(3), 365378.

Salo, J. (2017). Social media research in the industrial marketing field: Review of literature and future research directions. Industrial Marketing Management, 66, 115-129.

Schaltegger, S., \& Burritt, R. (2017). Contemporary Environmental Accounting: Issues, Concepts and Practice. Routledge.

Schultz, R. J., Schwepker Jr, C. H., \& Good, D. J. (2012). Social media usage: an investigation of B2B salespeople. American Journal of Business, 27(2), 174-194.

Stelzner, M. A. (2018). Social media marketing industry report. Retrieved from https:// www.socialmediaexaminer.com/wp-content/uploads/2018/05/Industry-Report-2018.pdf.

(Accessed date: 22.05.2019) 
Steyn, P., Salehi-Sangari, E., Pitt, L., Parent, M., \& Berthon, P. (2010). The Social Media Release as a public relations tool: Intentions to use among B2B bloggers. Public Relations Review, 36(1), 87-89.

Swani, K., Brown, B. P., \& Milne, G. R. (2014). Should tweets differ for B2B and B2C? An analysis of Fortune 500 companies' Twitter communications. Industrial Marketing Management, 43(5), 873-881.

Tajvidi, R., \& Karami, A. (2017). The effect of social media on firm performance. Computers in Human Behavior. https://doi.org/10.1016/j.chb.2017.09.026

Trainor, K. J., Andzulis, J. M., Rapp, A., \& Agnihotri, R. (2014). Social media technology usage and customer relationship performance: A capabilities-based examination of social CRM. Journal of Business Research, 67(6), 1201-1208.

Wolf, J., 2014. The relationship between sustainable supply chain management, stakeholder pressure and corporate sustainability performance. Journal of Business Ethics, 119(3), 317-328.

Xiong, Y., \& Zhang, J. (2014). Applying a life-oriented approach to evaluate the relationship between residential and travel behavior and quality of life based on an exhaustive CHAID approach. Procedia-Social and Behavioral Sciences, 138, 649-659.

Viglia, G., Pera, R., \& Bigné, E. (2018). The determinants of stakeholder engagement in digital platforms. Journal of Business Research, 89, 404-410.

Waara, N., Brundell-Freij, K., Risser, R., \& Ståhl, A. (2015). Feasible provision of targeted traveler information in public transportation: Segmentation based on functional limitations. Transportation Research Part A: Policy and Practice, 74, 164-173.

Wang, W. Y., Pauleen, D. J., \& Zhang, T. (2016). How social media applications affect B2B communication and improve business performance in SMEs. Industrial Marketing Management, 54, 4-14.

Williams, Z., Lueg, J. E., Hancock, T., \& Goffnett, S. P. (2018). Positioning through B2B carrier signals: Understanding how service quality is communicated via websites. Industrial Marketing Management.

Yang, C.-S., 2018. An analysis of institutional pressures, green supply chain management, and green performance in the container shipping context. Transportation Research Part D: Transport and Environment, 61, 246-260.

Yuen, K. F., \& Lim, J. M. (2016). Barriers to the implementation of strategic corporate social responsibility in shipping. The Asian Journal of Shipping and Logistics, 32(1), 49-57.

Yuen, K. F., \& Thai, V. (2017). Service quality appraisal: a study of interactions. Total Quality Management \& Business Excellence, 28(7-8), 730-745.

Yuen, K.F., Wang, X., Wong, Y.D., Zhou, Q., 2017. Antecedents and outcomes of sustainable shipping practices: The integration of stakeholder and behavioral theories. Transportation Research Part E: Logistics and Transportation Review, 108, 18-35. 
Yuen, K.F., Thai, V.V., Wong, Y.D., Wang, X., 2018. Interaction impacts of corporate social responsibility and service quality on shipping firms' performance. Transportation Research Part A: Policy and Practice 113, 397-409.

Zephoria. (2019). Top 10 Twitter Statistics -Updated April 2019. https://zephoria.com/twitterstatistics-top-ten/ (Accessed date: 21.05.2019)

Zhang, C. B., \& Lin, Y. H. (2015). Exploring interactive communication using social media. The Service Industries Journal, 35(11-12), 670-693. 